\title{
Saliva as a Non-Invasive Diagnostic Medium in Proteomics for Oral Squamous Cell Carcinoma Detection
}

\author{
Sajad Ahmad Buch ${ }^{1 *}$, Laxmikanth Chatra ${ }^{1}$ \\ ${ }^{1}$ Department of Oral Medicine \& Radiology, Yenepoya Dental College, Yenepoya (Deemed to be University), Mangalore, India
}

Corresponding Author: Sajad Ahmad Buch, Research Scholar, Department of Oral Medicine \& Radiology, Yenepoya Dental College, Yenepoya (Deemed to be University), Mangalore, India. Tel: +91-7899466346, Email: buchh.sajad@gmail.com

Received June 8, 2019; Accepted August 28, 2019; Online Published September 24, 2019

\begin{abstract}
Non-invasive diagnostic techniques are essential for the screening of oral cancer to reduce high mortality associated with it. The acknowledgement of various research advancements namely omic research and technological advances in the field of genomes has led to an increased use of saliva collection. Obtaining saliva for diagnosis is simple and non-invasive and is safe for both the health worker and the patient. It also permits easy and cost-effective storage methods. Around 3000 differently expressed proteins and peptides have been identified in various proteomic studies involving saliva, and hence, salivary proteomics is being progressively used for the early diagnosis of numerous diseases such as oral cancer, breast cancer, and several autoimmune disorders, among others. The salivary analysis riding on the recent advancements in technology, can prove to be of much more importance in near future.

Keywords: Biomarkers, Diagnosis, Oral Squamous Cell Carcinoma, Oral Potentially Malignant Disorders, Saliva

Citation: Buch SA, Chatra L. Saliva as a non-invasive diagnostic medium in proteomics for oral squamous cell carcinoma detection. Int J Med Rev. 2019;6(3):73-76. doi:10.29252/IJMR-060301.
\end{abstract}

\section{Introduction}

Oral cancer indicates all malignancies that arise from the lips, the oral cavity, and pharynx, with over 481000 new cases occurring worldwide annually and $90 \%$ of them being oral squamous cell carcinoma (OSCC) type. ${ }^{1}$ The most common type of head and neck malignant tumor, OSCC occupies the eighth spot in terms of frequencies among all cancer types worldwide. ${ }^{2}$ The standardized incidence rate of oral cancer in India stands at 12.6 per 100000 people. ${ }^{3}$ Major risk factors for OSCC include consumption of tobacco, alcohol intake and infections with human papilloma virus. ${ }^{4}$ Any form of tobacco use and excess intake of alcohol constitute the major risk factors of oral cancer. The symptoms of OSCC are presented in late stages and encountering the disease at late stages results in recurrences after treatment. The patients previously treated for OSCC, constitute another group that is at high risk for developing OSCC, because they are prone to recurring OSCC or a secondary lesion, as well as those who have history of some other malignancies other than OSCC. ${ }^{5}$ Oral cancer can occasionally be preceded by lesions of oral precancer which predominantly includes oral submucous fibrosis and leukoplakia. ${ }^{6}$ This distinction proposes a two-step development of oral cancer, i.e., the occurrence of a precursor initially followed by its transformation into cancer and is well substantiated. Currently identified oral potentially malignant disorders (OPMDs) include oral submucous fibrosis, erythroplakia, leukoplakia, oral lichen planus, palatal lesions associated with reverse smoking, actinic cheilitis and discoid lupus erythematosus. ${ }^{7}$ The prevention and timely recognition of such OPMDs not only favours a decreased rate of oral cancer but also improves the chances of survival in subjects developing oral cancer. ${ }^{8}$ The 5 -year survival rate of oral cancer has remained persistently at a dismal rate of around $50 \%$ despite major advances in its treatment. ${ }^{9}$ There has been a continuous effort towards developing novel diagnostic techniques, including new panels of OSSC biomarkers. As saliva is easy to obtain and can be collected non-invasively, it makes a great choice for this purpose.$^{10}$ Research exploring biomarkers in saliva for diagnosis of various cancers is being published routinely. ${ }^{11-14}$ The use of saliva for identifying oral cancer biomarkers has gained attention due to many reasons, one among them being its direct contact with the lesion. ${ }^{15}$ The use of saliva as liquid biopsy medium offers an edge over blood, as collection of saliva is safe, non-invasive, easy and cost-effective. ${ }^{16}$ Saliva constitutes a composite collection of cytokines, enzymes, hormones, antibodies and antimicrobial agents. ${ }^{17}$ The low molecular weight proteins constitute about $40 \%$ to $50 \%$ of proteins in the salivary glands. ${ }^{18}$ The chromosomes 4,12 and 20 contain the genes that encode for these proteins. ${ }^{19}$. The low molecular weight proteins in saliva were grouped into six main classes, namely, basic prolinerich proteins, glycosylated proline-rich proteins histatins, acidic proline-rich proteins, cystatins and statherins. ${ }^{20,21}$ Around 3000 differentially expressed peptides and proteins,

Copyright $\odot 2019$ The Author(s). This is an open-access article distributed under the terms of the Creative Commons Attribution License (http:// creativecommons.org/licenses/by/4.0), which permits unrestricted use, distribution, and reproduction in any medium, provided the original work is properly cited. 
a number of them being microbiological in origin have been characterized recently after analysing salivary proteome of humans. ${ }^{22}$ Salivaomics, omics procedures utilizing saliva, ${ }^{23}$ are at present confined to proteomics, transcriptomics, metabolomics, microbiomics and micro-RNA-omics. ${ }^{24}$ Proteomics is the new science of estimating the protein characteristics, their composition in the various organs and tissues of the body, at a previously inapproachable level. ${ }^{25}$ This makes it possible to make a significant advancement in the investigation of saliva, which owing to its accessibility and relation to blood has long gained the attention of researchers.

\section{Discussion}

OSCC with over 300000 fresh cases and about 145000 deaths annually throughout the world constitutes a high prevalence and morbidity. ${ }^{26}$ The high mortality and morbidity associated with OSCC, can still be attributed to its late diagnosis. A large number of OSCC cases, around two-thirds of all the cases are first encountered at an advanced stage. ${ }^{27}$ The early stages of OSCC have a fair prognosis with a cure rate of $80 \%$ in Stage I and $65 \%$ in Stage II. ${ }^{28}$ As most of the cases are diagnosed at late Stages of either III or IV, they have a poor survival 5 year rate of less than $50 \% .^{29}$ It is therefore of critical importance to detect OSCC early to reduce its morbidity and mortality. The incisional biopsy together with histopathological investigation constitutes the current gold standard in the diagnosis of OSCC. ${ }^{30}$ The scalpel biopsy although a gold standard for diagnosis, but being invasive, patients may show reluctance to accept the procedure. The expert clinical examination and histopathological investigation fails to detect the presence of early OSCC in most of the suspicious looking cases, thereby raising the need for the early detection of OSCC through improvement of diagnostic techniques. ${ }^{31}$

The effectiveness of saliva is still limited in clinical settings and requires additional research for its routine use as a diagnostic medium. The gold standard technique, biopsy, can confirm or even change the diagnosis of cancer with clear histopathological picture and shows grading of the disease ; it also reveals the type of cancer and any local invasion but is invasive in nature. The non-invasive and economical nature of saliva makes it an appealing diagnostic medium for various diseases. ${ }^{32}$ Although oral cavity is full of complexity owing to a large number of factors involved in maintaining its homeostasis, the use of saliva as a reliable diagnostic medium has been attempted and encouraging steps have been taken to prove its efficiency to detect protein biomarkers. ${ }^{22}$ Saliva is a very dilute body fluid which contains proteins, electrolytes and nitrogenous products. Among its protein content, the cystatins, $\alpha$-amylase, serum albumin, proline rich peptides and mucins are the most plentiful in Saliva. ${ }^{33}$ In addition to the most abundantly proteins found in saliva, the lesser ones have also been seen to have a definite diagnostic advantage in various diseases. ${ }^{34,35}$ The profiling of proteomes may be of significant importance to understand the pathogenesis of diseases and resultant identification of biomarkers, as proteins constitute the end products of gene information and act as final effectors for many cellular functions. ${ }^{36}$ The main methods to study salivary proteome include mass spectrometry (MS) and Two-dimensional electrophoresis (2D electrophoresis). These techniques have worked wonders in evaluation of salivary protein components, in combination with other techniques like drop electrophoresis, chromatography, and gel filtration. Several publications have summarized methodology bases of saliva associated proteomic research. ${ }^{37-39}$ The accumulation of data and the development of various tools like bioinformatics has resulted in the foundation of salivary proteome databases, Salvaomic database,${ }^{40}$ and SDXMART-BIOMART portal; data can be found here ranging from saliva proteome, peptidome, metabolome, and transcriptome. ${ }^{41}$ The first systemization of salivary proteins was published in 2008 after analysing saliva proteome for the first time. ${ }^{42}$

The serum proteomic studies in head and neck cancer (HNC) reveal that, proteins expressed differently can differentiate HNC patients from the control groups with a high degree of sensitivity (68\% to $83.3 \%),{ }^{43,44}$ and specificity(76\%-90\%). ${ }^{44,45}$ The studies employing salivary proteomics in a similar fashion have shown comparable reliability in detecting OSCC with a sensitivity of $90 \%$ and specificity of $83 \% .{ }^{46}$

Gleber Netto et al, concluded that salivary proteomics is valuable in the diagnosis of OSCC and OPMDs and such salivary analytes can diagnose OPMDs with high discriminatory powers enabling early detection of patients with high risk for OSCC development. ${ }^{47}$ Gallo et al, confirmed numerous significant modifications shown by salivary proteomes in OSCC patients; furthermore, few of these modifications are associated with nodal status, therefore, offering better prospects of understanding the process of carcinogenesis, and that these proteomes also help to discover useful potential biomarkers with dependable clinical utilities. ${ }^{36}$ Salivary proteomics is really worth for continuous evaluation until more discoveries are made in the struggle against OSCC. Chen et al, improved upon the workflow for salivary protein digestion and assessed quantification performance, strength and technical impediments in analysing clinical samples based on targeted proteomics using liquid chromatography (LC), multiple reaction monitoring (MRM) with MS detection (LCMRM/MS). ${ }^{48}$ They identified 56 proteins, previously seen associated with OSCC patients and found most of these significantly elevated in saliva samples from OSCC patients when compared with healthy controls. The developed platform assayed for the most highly multiplexed panel of salivary protein biomarkers, establishing the advantage of MRM in OSCC biomarker research.

Carnielli et al, combined the discovery and targeted proteomics methods to detect predictive proteomic signatures for OSCC patients. ${ }^{2}$ The discovery (proteomic analysis) phase of this study was performed on formalin-fixed paraffinembedded OSCC tissues and combined with clinical status of the participants, establishing several proteins(CSTB, NDRG1, LTA4H, PGK1, COL6A1, ITGAV, and MB), expressed in a distinct pattern between inner tumor and invasive tumor front, and thereby proposing a likely prognostic value of clinical and pathological analysis. This was followed by a targeted phase, with two separate approaches, through clinical significance 
and histochemical staining and the second one being selected reaction monitoring-mass spectrometry (SRM-MS) to evaluate the abundance of seven proteins in salivary samples of independent group of OSCC patients. The combination of COLA1-, LTA4H-, and CSTB- specific salivary peptides, in their study demonstrated the ability to discriminate between the nodal status of the patients with and without metastasis with a decent and better than the prediction value of single or grouped proteins.

Csosz et al, evaluated a non-invasive salivary diagnostic method for detection of useful biomarkers for early detection of OSCC by a Luminex-based multiplex kit for (VEGF, IL-6, IL-8, IL- $1 \alpha$, IL- $1 \beta$, TNF- $\alpha$ ) and SRM-based targeted proteomic method for (CD59, catalase, galectin-3-bindig protein, profilin-1,S100A9, CD44, thioredoxin and keratin-19). ${ }^{49}$ ELISA was used for validation of selected potential OSCC biomarkers, and revealed SA1009 and IL- 6 as useful OSCC biomarkers for detection and thus improving the diagnostic precision for OSCC. Shan et al, selected three proteomics salivary biomarkers for validation, S100A2, SLC3A2, and IL1RN from the 21 significantly altered proteins when OSCC was compared with both OPMDs and Controls. ${ }^{50}$ The findings of this study establishes the combination of SLC3A2, S100A2, and IL1RN as encouraging salivary biomarkers of OSCC. After additional validation the purely non-invasive salivary screening assay, may reveal better performance for early diagnosis of OSCC.

The medical diagnostic field is progressing by proteomic analyses because of its ability to reliably identify proteins, that were previously inaccessible. It is imperative to identify non-invasive sensitive and specific biomarkers for early diagnosis of OSCC. The ability to detect molecules in saliva, such as proteins, metabolites, DNA, coding and noncoding RNAs indicates that, saliva has enormous value in the early diagnosis of OSCC..$^{51}$

\section{Conclusions}

Saliva has been in use since a long time for routine clinical laboratory practices notable among them being evaluation of hormones, cortisol and secretory antibodies. As a diagnostic medium it enjoys several advantages from, undemanding collection technique, safer to handle, non-invasive and being able to be shipped and stored in an easy and cost-effective approach. Salivary proteome has recently been explored for understanding the efficiency of saliva as a diagnostic medium. The various proteomics studies mentioned in this review favour diagnostic role of saliva in discriminating OSSC from oral precancer and healthy controls, thereby providing effective means of monitoring and arresting the development of oral cancer. Various attempts have been made to develop rapid salivary methods of OSCC detection with optimistic outcome. Today, proteomic technologies are extremely complex, expensive and of limited accessibility. The time is not far when further continuous research in -omics related studies will produce simple, reasonably priced and ergonomic instruments, that can be effectively applied to saliva for diagnosing OSCC at a rapid, cost-effective and noninvasion fashion.

\section{Authors' Contributions}

SAB performed literature survey and compiled the manuscript. LC supervised the manuscript, generation and performed critical analysis.

\section{Conflict of Interest Disclosures}

The authors declare they have no conflicts of interest.

\section{Acknowledgements}

We would like to thank Dr. Vinitha Ramanath Pai, Deputy Director, Centre for $\mathrm{PhD}$ and Professor T.S. Keshava Prasad, Deputy Director, Centre for Systems Biology and Molecular Medicine, Yenepoya (Deemed to be University), Mangalore-Karnataka, India, for their constant support and encouragement.

\section{References}

1. Markopoulos AK, Michailidou EZ, Tzimagiorgis G. Salivary markers for oral cancer detection. Open Dent J. 2010;4:172-178. doi:10.2174/1874210601004010172.

2. Carnielli CM, Macedo CCS, De Rossi T, et al. Combining discovery and targeted proteomics reveals a prognostic signature in oral cancer. Nat Commun. 2018;9(1):3598. doi:10.1038/s41467-01805696-2.

3. Byakodi R, Byakodi S, Hiremath S, et al. Oral cancer in India: an epidemiologic and clinical review. J Community Health. 2012;37(2):316-319. doi:10.1007/s10900-011-9447-6.

4. Llewellyn CD, Johnson NW, Warnakulasuriya KA. Risk factors for squamous cell carcinoma of the oral cavity in young people--a comprehensive literature review. Oral Oncol. 2001;37(5):401418. doi:10.1016/S1368-8375(00)00135-4.

5. Nagler RM, Kerner H, Laufer D, Ben-Eliezer S, Minkov I, BenItzhak O. Squamous cell carcinoma of the tongue: the prevalence and prognostic roles of $\mathrm{p} 53, \mathrm{Bcl}-2, \mathrm{c}-\mathrm{erbB}-2$ and apoptotic rate as related to clinical and pathological characteristics in a retrospective study. Cancer Lett. 2002;186(2):137-150. doi:10.1016/s03043835(02)00265-3.

6. Elango JK, Gangadharan P, Sumithra S, Kuriakose MA. Trends of head and neck cancers in urban and rural India. Asian Pac J Cancer Prev. 2006;7(1):108-112.

7. Liu D, Zhao X, Zeng X, Dan H, Chen Q. Non-Invasive Techniques for Detection and Diagnosis of Oral Potentially Malignant Disorders. Tohoku J Exp Med. 2016;238(2):165-177. doi:10.1620/ tjem.238.165.

8. Sarode SC, Sarode GS, Karmarkar S. Early detection of oral cancer: detector lies within. Oral Oncol. 2012;48(3):193-194. doi:10.1016/j.oraloncology.2011.11.018.

9. Kawahara R, Bollinger JG, Rivera C, et al. A targeted proteomic strategy for the measurement of oral cancer candidate biomarkers in human saliva. Proteomics. 2016;16(1):159-173. doi:10.1002/ pmic.201500224.

10. Bonne NJ, Wong DT. Salivary biomarker development using genomic, proteomic and metabolomic approaches. Genome Med. 2012;4(10):82. doi:10.1186/gm383.

11. Porto-Mascarenhas EC, Assad DX, Chardin H, et al. Salivary biomarkers in the diagnosis of breast cancer: A review. Crit Rev Oncol Hematol. 2017;110:62-73. doi:10.1016/j. critrevonc.2016.12.009

12. Cheng YS, Rees T, Wright J. A review of research on salivary biomarkers for oral cancer detection. Clin Transl Med. 2014;3(1):3. doi:10.1186/2001-1326-3-3.

13. Humeau M, Vignolle-Vidoni A, Sicard F, et al. Salivary MicroRNA in Pancreatic Cancer Patients. PLoS One. 2015;10(6):e0130996. doi:10.1371/journal.pone.0130996.

14. Xiao H, Zhang $Y, K i m$, et al. Differential Proteomic Analysis of Human Saliva using Tandem Mass Tags Quantification for 
Gastric Cancer Detection. Sci Rep. 2016;6:22165. doi:10.1038/ srep22165.

15. Shah FD, Begum R, Vajaria BN, et al. A review on salivary genomics and proteomics biomarkers in oral cancer. Indian J Clin Biochem. 2011;26(4):326-334. doi:10.1007/s12291-011-0149-8.

16. Yoshizawa JM, Schafer CA, Schafer JJ, Farrell JJ, Paster BJ, Wong DT. Salivary biomarkers: toward future clinical and diagnostic utilities. Clin Microbiol Rev. 2013;26(4):781-791. doi:10.1128/ cmr.00021-13.

17. Spielmann N, Wong DT. Saliva: diagnostics and therapeutic perspectives. Oral Dis. 2011;17(4):345-354. doi:10.1111/j.16010825.2010.01773.x.

18. Amado FM, Vitorino RM, Domingues PM, Lobo MJ, Duarte JA. Analysis of the human saliva proteome. Expert Rev Proteomics. 2005;2(4):521-539. doi:10.1586/14789450.2.4.521.

19. Huq NL, Cross KJ, Ung M, et al. A review of the salivary proteome and peptidome and saliva-derived peptide therapeutics. Int J Pept Res Ther. 2007;13(4):547-564. doi:10.1007/s10989-007-9109-9.

20. Schlesinger DH, Hay DI, Levine MJ. Complete primary structure of statherin, a potent inhibitor of calcium phosphate precipitation, from the saliva of the monkey, Macaca arctoides. Int J Pept Protein Res. 1989;34(5):374-380. doi:10.1111/j.1399-3011.1989. tb00705.x.

21. Amado F, Lobo MJ, Domingues P, Duarte JA, Vitorino R. Salivary peptidomics. Expert Rev Proteomics. 2010;7(5):709-721. doi:10.1586/epr.10.48

22. Grassl N, Kulak NA, Pichler G, et al. Ultra-deep and quantitative saliva proteome reveals dynamics of the oral microbiome. Genome Med. 2016;8(1):44. doi:10.1186/s13073-016-0293-0.

23. Tasoulas J, Patsouris E, Giaginis C, Theocharis S. Salivaomics for oral diseases biomarkers detection. Expert Rev Mol Diagn. 2016;16(3):285-295. doi:10.1586/14737159.2016.1133296.

24. Kaczor-Urbanowicz KE, Martin Carreras-Presas C, Aro K, Tu M, Garcia-Godoy F, Wong DT. Saliva diagnostics - Current views and directions. Exp Biol Med (Maywood). 2017;242(5):459-472. doi:10.1177/1535370216681550.

25. Kolesov SA, Fedulova EN, Lavrova AE. Characteristics of human saliva proteome and peptidome. Hum Physiol. 2016;42(4):463468. doi:10.1134/s0362119716040058.

26. Ferlay J, Soerjomataram I, Dikshit R, et al. Cancer incidence and mortality worldwide: sources, methods and major patterns in GLOBOCAN 2012. Int J Cancer. 2015;136(5):E359-386. doi:10.1002/ijc.29210.

27. McGurk M, Chan C, Jones J, O'Regan E, Sherriff M. Delay in diagnosis and its effect on outcome in head and neck cancer. Br J Oral Maxillofac Surg. 2005;43(4):281-284. doi:10.1016/j. bjoms.2004.01.016.

28. Guneri P, Epstein JB. Late stage diagnosis of oral cancer: components and possible solutions. Oral Oncol. 2014;50(12):1131-1136. doi:10.1016/j.oraloncology.2014.09.005.

29. Viet CT, Schmidt BL. Biologic mechanisms of oral cancer pain and implications for clinical therapy. J Dent Res. 2012;91(5):447-453. doi:10.1177/0022034511424156.

30. Macey R, Walsh T, Brocklehurst P, et al. Diagnostic tests for oral cancer and potentially malignant disorders in patients presenting with clinically evident lesions. Cochrane Database Syst Rev. 2015(5):CD010276. doi:10.1002/14651858.CD010276.pub2.

31. Zhang H, Dziegielewski PT, Biron VL, et al. Survival outcomes of patients with advanced oral cavity squamous cell carcinoma treated with multimodal therapy: a multi-institutional analysis. J Otolaryngol Head Neck Surg. 2013;42:30. doi:10.1186/19160216-42-30.

32. Shpitzer T, Hamzany Y, Bahar G, et al. Salivary analysis of oral cancer biomarkers. Br J Cancer. 2009;101(7):1194-1198. doi:10.1038/sj.bjc.6605290.

33. CsőszzÉ, Kalló G, Márkus B, Deák E, CsutakA, Tőzsér J. Quantitative body fluid proteomics in medicine - A focus on minimal invasiveness. J Proteomics. 2017;153:30-43. doi:10.1016/j. jprot.2016.08.009

34. Thumbigere-Math V, Michalowicz BS, de Jong EP, et al. Salivary proteomics in bisphosphonate-related osteonecrosis of the jaw. Oral Dis. 2015;21(1):46-56. doi:10.1111/odi.12204.

35. Streckfus CF, Mayorga-Wark O, Arreola D, Edwards C, Bigler L, Dubinsky WP. Breast cancer related proteins are present in saliva and are modulated secondary to ductal carcinoma in situ of the breast. Cancer Invest. 2008;26(2):159-167. doi:10.1080/07357900701783883.

36. Gallo C, Ciavarella D, Santarelli A, et al. Potential Salivary Proteomic Markers of Oral Squamous Cell Carcinoma. Cancer Genomics Proteomics. 2016;13(1):55-61.

37. Al-Tarawneh SK, Border MB, Dibble CF, Bencharit S. Defining salivary biomarkers using mass spectrometry-based proteomics: a systematic review. Omics. 2011;15(6):353-361. doi:10.1089/ omi.2010.0134.

38. Hu S, Loo JA, Wong DT. Human saliva proteome analysis and disease biomarker discovery. Expert Rev Proteomics. 2007;4(4):531-538. doi:10.1586/14789450.4.4.531.

39. Castagnola M, Cabras T, lavarone F, et al. Top-down platform for deciphering the human salivary proteome. J Matern Fetal Neonatal Med. 2012;25(Suppl 5):27-43. doi:10.3109/14767058.2012.714 647.

40. Zhang L, Xiao H, Wong DT. Salivary biomarkers for clinical applications. Mol Diagn Ther. 2009;13(4):245-259. doi:10.2165/11317160-000000000-00000.

41. Ai JY, Smith B, Wong DT. Bioinformatics advances in saliva diagnostics. Int J Oral Sci. 2012;4(2):85-87. doi:10.1038/ ijos.2012.26.

42. Choi M. Saliva diagnostics integrate dentistry into general and preventive health care. Int J Prosthodont. 2010;23(3):189.

43. Soltys SG, Le QT, Shi G, Tibshirani R, Giaccia AJ, Koong AC. The use of plasma surface-enhanced laser desorption/ionization time-of-flight mass spectrometry proteomic patterns for detection of head and neck squamous cell cancers. Clin Cancer Res. 2004;10(14):4806-4812. doi:10.1158/1078-0432.ccr-03-0469.

44. Wadsworth JT, Somers KD, Stack BC Jr, et al. Identification of patients with head and neck cancer using serum protein profiles. Arch Otolaryngol Head Neck Surg. 2004;130(1):98-104. doi:10.1001/archotol.130.1.98.

45. Gourin CG, Xia ZS, Han Y, et al. Serum protein profile analysis in patients with head and neck squamous cell carcinoma. Arch Otolaryngol Head Neck Surg. 2006;132(4):390-397. doi:10.1001/ archotol.132.4.390.

46. Hu S, Arellano M, Boontheung P, et al. Salivary proteomics for oral cancer biomarker discovery. Clin Cancer Res. 2008;14(19):62466252. doi:10.1158/1078-0432.ccr-07-5037.

47. Gleber-Netto FO, Yakob M, Li F, et al. Salivary Biomarkers for Detection of Oral Squamous Cell Carcinoma in a Taiwanese Population. Clin Cancer Res. 2016;22(13):3340-3347. doi:10.1158/1078-0432.ccr-15-1761.

48. Chen YT, Chen HW, Wu CF, et al. Development of a Multiplexed Liquid Chromatography Multiple-Reaction-Monitoring Mass Spectrometry (LC-MRM/MS) Method for Evaluation of Salivary Proteins as Oral Cancer Biomarkers. Mol Cell Proteomics. 2017;16(5):799-811. doi:10.1074/mcp.M116.064758.

49. Csősz É, Lábiscsák P, Kalló G, et al. Proteomics investigation of OSCC-specific salivary biomarkers in a Hungarian population highlights the importance of identification of population-tailored biomarkers. PLoS One. 2017;12(5):e0177282. doi:10.1371/ journal.pone.0177282.

50. Shan J, Sun Z, Yang J, et al. Discovery and preclinical validation of proteomic biomarkers in saliva for early detection of oral squamous cell carcinomas. Oral Dis. 2019;25(1):97-107. doi:10.1111/odi.12971.

51. Siravegna G, Marsoni S, Siena S, Bardelli A. Integrating liquid biopsies into the management of cancer. Nat Rev Clin Oncol. 2017;14(9):531-548. doi:10.1038/nrclinonc.2017.14 